\title{
Compact high-resolution retarding field energy analyzer for space-charge-dominated electron beams
}

\author{
Y. Zou, Y. Cui, V. Yun, A. Valfells, R. A. Kishek, S. Bernal, I. Haber, M. Reiser, and P. G. O’Shea \\ Institute for Research in Electronics and Applied Physics, University of Maryland, College Park, Maryland 20742 \\ J. G. Wang \\ SNS, Oak Ridge National Laboratory, Oak Ridge, Tennessee 37831 \\ (Received 22 April 2002; published 5 July 2002)
}

\begin{abstract}
We have developed a compact high-resolution retarding field energy analyzer for measuring the energy spread of space-charge-dominated electron beams. This energy analyzer has a cylindrical electrode to overcome the defocusing effects due to space-charge forces, beam trajectories, aperture effect, etc. The device provides excellent spatial and temporal information on the beam energy spread. Single-particle simulation shows that this energy analyzer has very good resolution for low-energy electron beams of several kilovolts and with large divergence angles. The energy analyzer has been tested with $2.5 \mathrm{keV}$, $60 \mathrm{~mA}$ electron beams. The measured energy spread is also compared with the theoretical calculations taking into account two main energy spread sources, namely, the Boersch effect and the longitudinallongitudinal relaxation.
\end{abstract}

DOI: $10.1103 /$ PhysRevSTAB.5.072801

PACS numbers: 29.30.Dn, 29.27.Fh, 29.17.+w

\section{INTRODUCTION}

Advanced particle accelerators for heavy-ion inertial fusion, high-energy colliders, free electron lasers, and other applications require high-quality, intense beams, which can be focused onto a small diameter target. For such applications, the beams must have very low emittance and low-energy spread. While there have been many experimental and theoretical investigations of emittance growth in intense beams, very little work exists on the sources and evolution of energy spread in such beams. At the University of Maryland, various experiments have been carried out to study space-charge-dominated electron beams. Some of these experiments, such as the resistive-wall instability experiment [1] and the University of Maryland Electron Ring (UMER) [2] currently being constructed, require knowledge of the beam's energy spread. The initial energy spread of the beams from the gun with a thermionic emitter and the growth of this energy spread in the transport line must be measured with high precision. For these applications, a high-resolution energy analyzer is necessary. The work described here deals with measurements of beams from thermionic sources only; however, in the near future we plan to extend our exploration to include photoemitters also.

In order to gain a full understanding of the evolution of the energy spread, we require a device that has excellent spatial and temporal resolution such that we can develop a 3D map of the energy spread in the beam. This is important because the energy spread may vary from head to tail as well as transversely in the beam bunch. Because of its simplicity and high signal-to-noise ratio output, the retarding field energy analyzer becomes a natural choice for low-energy electron beams. In parallel plate structures, however, space-charge forces, beam trajectories, mechani- cal misalignment, beam mismatching, etc., often lead to poor resolution of the energy measurement. In this paper, we report on the design and testing results of a cylindrical retarding field energy analyzer, which has greatly improved the resolution compared to a parallel plate energy analyzer used previously in our experiments. In the following sections, we first discuss the theory of the energy spread source in the space-charge-dominated beams and then we will describe the design, simulation, and beam tests of the new retarding field energy analyzer.

\section{SOURCES OF BEAM ENERGY SPREAD FROM A THERMIONIC GUN}

There are many sources contributing to the longitudinal energy spread for an electron beam emitted from a thermionic electron gun. The two dominant sources for the energy spread are the Boersch effect [3] and the longitudinallongitudinal relaxation effect [4]. The Boersch effect is due to the energy transfer from the transverse direction to the longitudinal direction. The longitudinal-longitudinal effect is due to the fast beam acceleration in the electron gun and therefore the energy transfer from potential energy to the thermal kinetic energy. Here we outline the calculations of the beam energy spread due to these two sources.

First, consider the Boersch effect following Ref. [5]. When the beam is emitted from the cathode, it has an inherent energy spread due to the cathode temperature. The rms beam energy spread is related to the cathode temperature by

$$
\Delta E_{\mathrm{rms}}=m v_{\mathrm{rms}}^{2}=k_{B} T .
$$

Here $v_{\text {rms }}$ is the rms thermal velocity, $k_{B}$ is the Boltzmann constant, and $T$ is the beam temperature. For a typical gun with cathode temperature of $1100{ }^{\circ} \mathrm{C}$, the corresponding 
thermal energy is around $0.1 \mathrm{eV}$. For beams emitted from photocathodes, this number may be larger, perhaps a few tenths of an electron volt.

When the beams are accelerated by a static electric field, every particle gains the same longitudinal kinetic energy. Consider two particles initially having an energy difference $\Delta E$; after acceleration they will still have the same amount of energy difference. However, their velocity difference and, therefore, the temperature of the beams will change. It can be shown that the beam temperature $T_{\| f}$ after acceleration is given by [5]

$$
k_{B} T_{\| f}=\left(k_{B} T_{\| i}\right)^{2} /\left(2 q V_{0}\right) .
$$

Here $T_{\| i}$ is the initial beam temperature, which is isotropic (i.e., $T_{\| i}=T_{\perp i}=T_{i}$ ) before the acceleration and $V_{0}$ is the accelerating voltage. This effect is known as the longitudinal cooling effect due to the acceleration.

Note that the transverse temperature $T_{\perp f}$ is the same as the initial temperature since the acceleration acts only in the longitudinal direction. The longitudinal temperature, which is determined by Eq. (2), becomes negligibly small compared to the transverse temperature. The beam is now in an extremely anisotropic state $\left(T_{\| f} \ll T_{\perp f}\right)$. Coulomb collisions and other processes will try to force the beam velocity distribution into thermal equilibrium. The resulting beam longitudinal energy spread will increase. This thermal relaxation process is referred to as the Boersch effect.

The transverse-longitudinal temperature relaxation effect is very complicated in a real beam. For a simple case in which the beam propagates through a smooth focusing channel and has a constant radius, the temperature relaxation can be described by the following equations [5]:

$$
T_{\perp}-2 / 3 T_{\perp i}\left(1+0.5 e^{-3 t / \tau_{\text {eff }}}\right),
$$

and

$$
T_{\|}=2 / 3 T_{\perp i}\left(1-e^{-3 t / \tau_{\mathrm{eff}}}\right),
$$

where $\tau_{\text {eff }}$ is the effective temperature relaxation time and is given by

$$
\tau_{\text {eff }}=4.44 \times 10^{20}\left(k_{B} T_{\text {eff }} / m c^{2}\right)^{3 / 2} /(n \ln \Lambda) .
$$

Here $T_{\text {eff }}$ is related to $T_{\perp i}$ by

$$
k_{B} T_{\text {eff }}=0.373 k_{B} T_{\perp i} .
$$

$\ln \Lambda$ is the Coulomb logarithm and is obtained from

$$
\ln \Lambda=\ln \left[5.66 \times 10^{21}\left(k_{B} T / m c^{2}\right)^{3 / 2} n^{-1 / 2}\right] .
$$

In the above equation, $n$ is the particle density given by

$$
n=I /\left(e a^{2} \pi v\right),
$$

where $I$ is the beam current, $a$ is the beam radius, and $v$ is the beam velocity. From Eq. (4), we find that the final longitudinal and transverse temperature will reach the equilibrium state at two thirds of the initial transverse temperature. The time it takes to reach equilibrium is about $\tau_{\text {eff }}$.
The energy transfer from the transverse direction to the longitudinal direction due to the Boersch effect can be suppressed by applying a strong axial magnetic field. However, even if this energy transfer is suppressed, the final beam energy spread is still found to be larger than what is predicted by the transverse-longitudinal cooling effect. The reason is that the Coulomb collisions in addition to the Boersch effect cause another temperature relaxation called longitudinal-longitudinal relaxation [4]. If the acceleration is fast (nonadiabatic) compared to the period of the electron beam plasma oscillation period, the initial state of relative high density and slow motion of electrons relative to each other is preserved during the acceleration process. Coulomb collisions tend to relax this nonequilibrium state into a more homogenous state and result in energy spread increase. The criteria for the adiabatic condition is determined by a dimensionless parameter $\lambda$, which compares the cooling time $\left(-1 / T \times d t_{\|} / d t\right)^{-1}$ with the plasma pe$\operatorname{riod} \omega_{p}^{-1}$ and is given by the formula [4]

$$
\lambda=-1 /\left(\omega_{p} T\right) \frac{d T_{\|}}{d t} .
$$

If $\lambda<1$, then the acceleration is adiabatic. $\lambda$ depends on the acceleration structure. It is interesting to note that a thermal electron gun with Pierce geometry has $\lambda$ equal to $2^{3 / 2}$, a nonadiabatic case [4], which applies to our experiment.

Combining both the transverse-longitudinal Boersch effect and the longitudinal-longitudinal relaxation effect, the final beam energy spread can be expressed as

$$
\Delta \tilde{E}_{\| f}=\left[\frac{1}{\pi \varepsilon_{0}} q n^{1 / 3} q V_{0}+2 q V_{0} k_{B} T_{\|}\right]^{1 / 2} .
$$

Here $\Delta \tilde{E}_{\| f}$ is the rms energy spread after acceleration and subsequent beam propagation; $q V_{0}$ is the beam energy and $T_{\|}$is an increasing function of time or distance of beam propagation, which can be calculated from Eq. (4) (Boersch effect). All the beam energies are in units of eV; $n$ is the beam density and $q$ is the electron charge. The first term in the bracket corresponds to the longitudinallongitudinal effect, and the second term is the transverse-longitudinal (Boersch) effect. The longitudinallongitudinal effect is stronger in the initial acceleration and propagation phase when there is a density and longitudinal temperature gradient. The energy spread due to the Boersch effect, on the other hand, increases monotonically until the longitudinal temperature reaches equilibrium. After a certain time of propagation, the Boersch effect will become the dominant source of the energy spread.

\section{DESIGN AND SIMULATION OF THE RETARDING FIELD ENERGY ANALYZER}

There are several types of retarding field energy analyzers as described in Ref. [6], which reviews retarding field analyzers with geometries of the parallel plate, spherical condenser, and Faraday cage [6]. The most commonly 
used one is the parallel plate energy analyzer, which consists of two parallel plates. This kind of device has been used for the UMER project in the past experiments [7]. The first plate is the grounding plate and the second one is biased to a negative high voltage for retardation of electron beams. Only those particles that have a longitudinal kinetic energy higher than the retarding voltage can pass through the second electrode and introduce a current signal at the collector. This structure has good resolution only for a beam with trajectories parallel to the axis of the energy analyzer. In practice, due to space-charge forces, beam emittance, and misalignment, etc., the beam particles always have finite divergence angles. The diverging particle trajectories lead to poor resolution. Our modification to this structure, in which the retarding voltage plate is replaced with a cylindrical electrode, has significantly improved the energy resolution [8]. As will be shown below, the computer simulation predicts that the resolution of the cylindrical energy analyzer is $0.6 \mathrm{eV}$ for a $2.5 \mathrm{keV}$ beam, corresponding to a relative resolution of $0.024 \%$. Another feature of this retarding field energy analyzer is its compact size. With a length of $4.3 \mathrm{~cm}$ and a diameter of $5.1 \mathrm{~cm}$, this analyzer can be easily inserted at any place in the beam line.

Figure 1 shows the schematic of the energy analyzer. The electron beam comes from the left. The first plate is a grounded plate with a circular aperture through which a small beamlet passes into the high voltage region. Because the energy analyzer samples a small fraction of the full beam, the beamlet in the energy analyzer is much less space charge dominated than the whole beam. The aperture is covered with a metal mesh to ensure the uniformity of electrical fields near the grounded plate. The high voltage cylinder is supported by two Macor rings and has a feedthrough to connect to the external high voltage source. The rear end of the high voltage cylinder is also covered with a wire mesh. Behind the high-voltage cylinder is a copper collector, from which the current signal is picked up by a $50 \Omega$ BNC connector. The whole structure is shielded

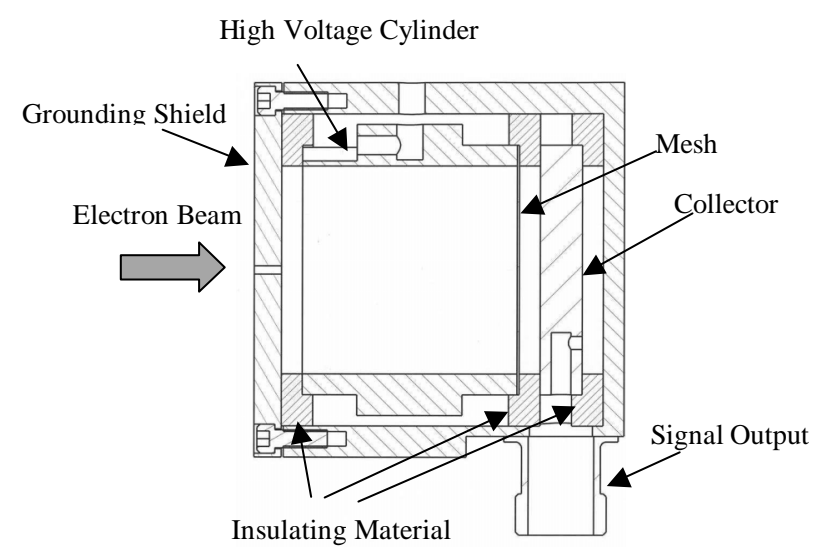

FIG. 1. Schematic of the energy analyzer with cylindrical high voltage electrode. The length is $4.3 \mathrm{~cm}$ and the diameter is $5.1 \mathrm{~cm}$. The aperture diameter is $1 \mathrm{~mm}$. to ensure good signal-to-noise ratio. Because of the tight space of the diagnostic chamber to hold the energy analyzer, the collector is a single plate instead of a regular Faraday cup structure. The secondary electron emission is suppressed due to the reverse electric field on the surface of the collector.

A computer code called SIMION was used to simulate the performance of the energy analyzer. SIMION is a PC-based charged particle optics simulation program. A wire mesh can also be incorporated in the program. Figure 2 depicts the simulated equipotential lines and the typical beam trajectories in the energy analyzer. The curved equipotential lines help to focus the beam. Figure 3 illustrates beam trajectories in this energy analyzer at different retarding voltages. The apparent voids in the beam in each figure are due to the limited number of beamlets in the simulation. The depicted beam is monoenergetic at an energy of $2.5 \mathrm{keV}$. The initial beam radius is $0.5 \mathrm{~mm}$, which is determined by the size of the aperture in the front plate. The divergence angle of the beam is $5^{\circ}$. Figure 3(a) is for a retarding voltage of $2.4994 \mathrm{kV}$, in which case all beam particles can pass through and appear as the current signal at the collector. Figure 3(b) is for a retarding voltage of $2.4997 \mathrm{kV}$, where some particles are reflected back while some particles still can pass through, depending on the divergence of their trajectories. When the retarding voltage is raised to $2.500 \mathrm{kV}$, all particles are reflected and the analyzer acts as a mirror, as shown in Fig. 3(c). Figure 4 shows the simulated energy analyzer output versus the retarding voltage. The triangular points are the simulated data points and the curves connecting the simulated data points are simple straight lines for illustrating purposes.

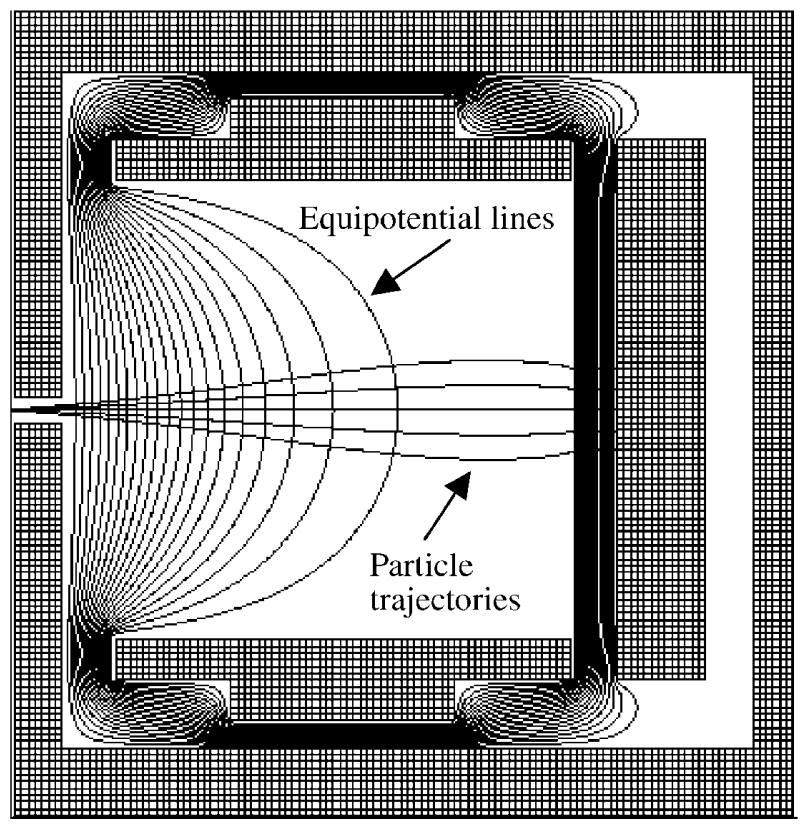

FIG. 2. Equipotential lines and typical beam trajectories in the energy analyzer. 
(a) Retarding voltage is $2.4994 \mathrm{kV}$

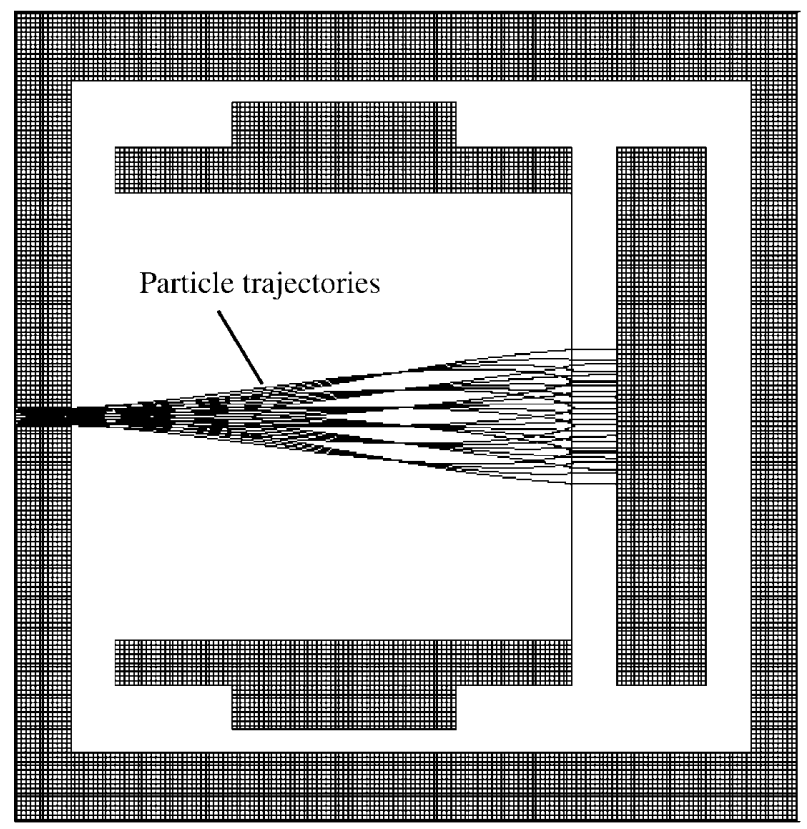

(b) Retarding voltage is $2.4997 \mathrm{kV}$

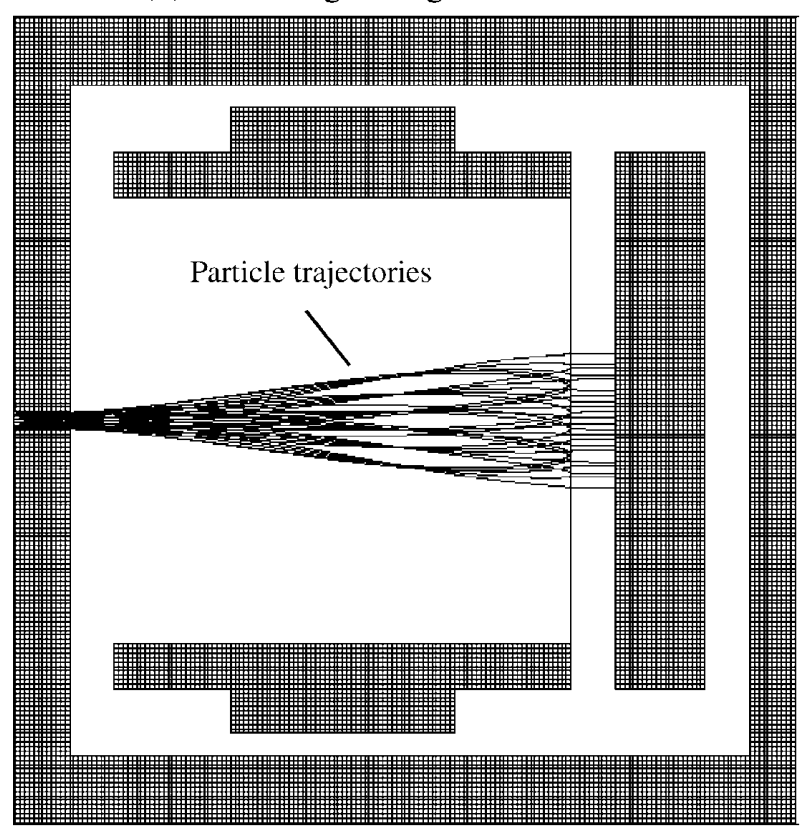

(c) Retarding voltage is $2.500 \mathrm{kV}$

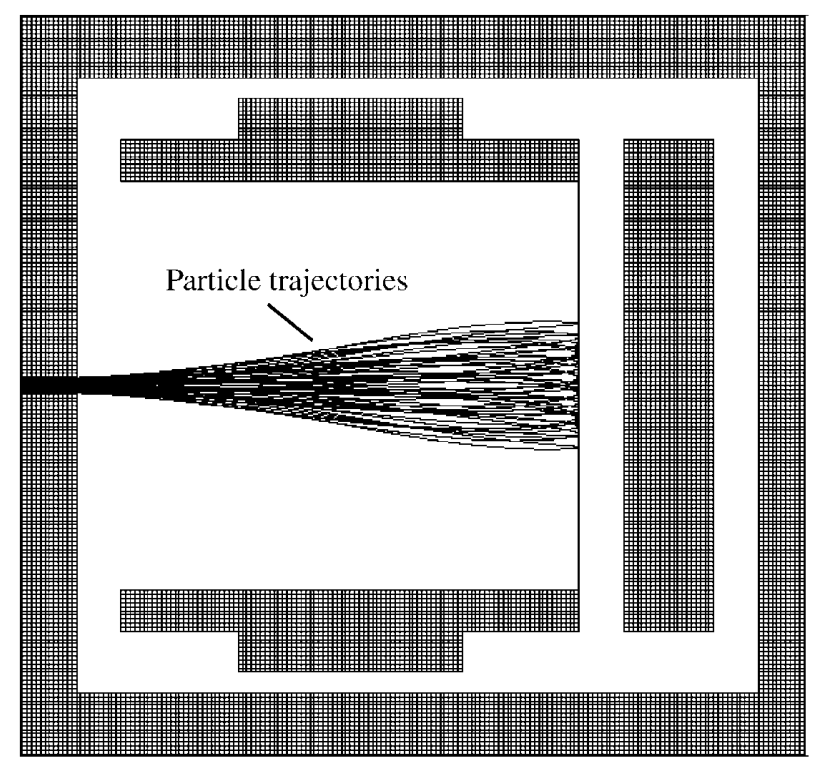

FIG. 3. Particle trajectories at different retarding voltages.

The ideal response of the energy analyzer has a sharp cutoff at $2.5 \mathrm{kV}$. For this energy analyzer, the curve starts to drop off at $2.4994 \mathrm{kV}$, giving the resolution of the device of around $0.6 \mathrm{eV}$. The curve is totally cut off at $2.5 \mathrm{kV}$, which corresponds to the total beam energy.

To compare the performance of the cylindrical analyzer to that of the parallel plate energy analyzer, we also simulated the performance of a parallel plate energy analyzer with the same beam parameters. The response of the parallel plate energy analyzer is also plotted in Fig. 4. The figure shows that the resolution of the parallel plate energy analyzer is around $20 \mathrm{eV}$. From this comparison, we can see the significant improvement of the resolution of the cylindrical energy analyzer compared to that of a parallel plate structure.

It is interesting to note that the performance of the device depends on the various beam parameters, for example, the beam kinetic energy $E_{k}$. Figure 5 depicts the simulations of the performance of the cylindrical energy analyzer with beam energies of 2.5, 5.5, and $10 \mathrm{keV}$. The resolution 


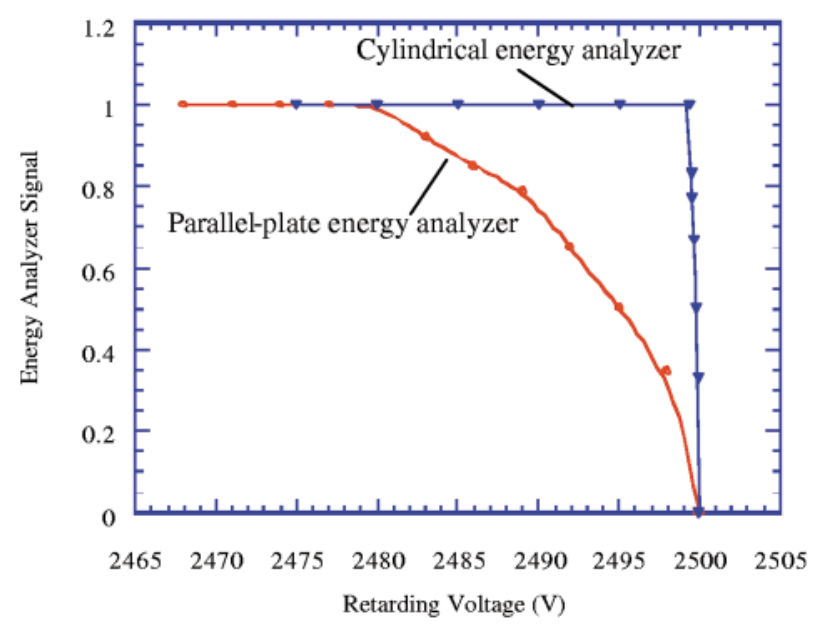

FIG. 4. (Color) Simulation shows that, for the beam with divergence angle of $5^{\circ}$, the cylindrical energy analyzer has a resolution of $0.6 \mathrm{eV}$, while the parallel plate counterpart has a resolution of $20 \mathrm{eV}$.

at $10 \mathrm{keV}$ is around $2.2 \mathrm{eV}$, worse than the $2.5 \mathrm{keV}$ case. The reason that the energy analyzer has poor resolution at higher beam energy is as follows. The Euler trajectory equations in a conserved system can be expressed as [5]

$$
r^{\prime \prime}=\left(1+r^{\prime 2}\right)\left(\partial \phi / \partial r-r^{\prime} \partial \phi / \partial z\right) /(2 \phi) .
$$

Here $\phi$ is the electrostatic potential at a certain position corresponding to the particle's kinetic energy at that point. It can be shown from this equation that the radial position $r(z)$ and the slope $r^{\prime}(z)=v_{r} / v_{z}$ of a particle trajectory in an axisymmetric electrostatic field, such as that in our energy analyzer, remain the same at any given distance $z$ along the axis if the electrostatic potentials are changed by the same factor at every point and the beam

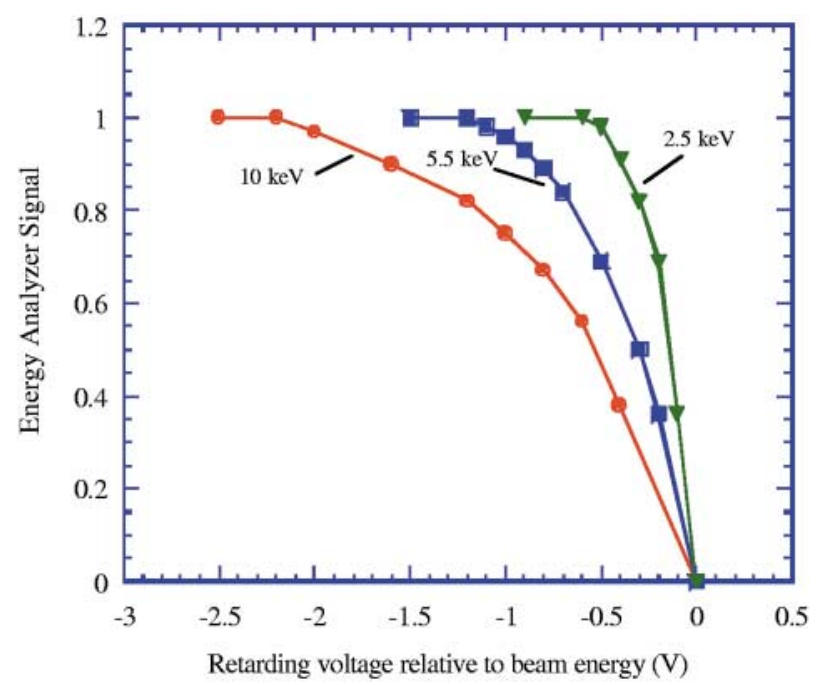

FIG. 5. (Color) Energy analyzer response curves to a monoenergetic beam at different beam energies of $2.5,5.5$, and $10 \mathrm{keV}$, respectively. initial conditions $\left[r\left(z_{0}\right)\right.$ and $\left.r^{\prime}\left(z_{0}\right)\right]$ remain the same. Since $v_{r} / v_{z}=\tan (\theta)$, then the difference between the longitudinal kinetic energy $E_{z}$ and the total kinetic energy $E_{k}$, $\Delta E$, is given by

$$
\Delta E=E_{k} \sin ^{2}(\theta) .
$$

Since $\theta$ remains constant under the above scaling rules as $E_{k}$ increases, it follows that the resolution of the electrostatic energy analyzer, $\Delta E$, is proportional to $E_{k}$. In another words, if we define the relative resolution $\Delta E / E$, then it is a constant independent of the total beam energy.

\section{BEAM TEST OF THE ENERGY ANALYZER}

The experimental setup, as shown in Fig. 6, consists of a gridded thermionic electron gun, three solenoidal magnetic lenses, and a diagnostic chamber. The energy analyzer is in the diagnostic chamber located between the second and the third solenoids. The third solenoid is after the energy analyzer and consequently, it has no effect for the beam trajectories in the energy analyzer. The distances of the first two solenoids and the energy analyzer from the electron gun are 9.1, 21.6, and $33.2 \mathrm{~cm}$, respectively. The solenoidal magnetic field extends less than $10 \mathrm{~cm}$ from the center, so there is practically no magnetic field inside the energy analyzer. Figure 7 depicts a typical beam envelope in the transport line calculated from the $\mathrm{K}-\mathrm{V}$ envelope equation. We can see that the energy analyzer is located at the waist of the beam. The energy analyzer can be aligned with respect to the beam by a linear and rotation feedthrough.

Figure 8 is a typical output signal from the energy analyzer for a beam with an energy of $5 \mathrm{keV}$ and a pulse length of $100 \mathrm{~ns}$. From the signal, we can see that this energy analyzer has a very fast rise time $(\sim 2 \mathrm{~ns})$ and can reproduce the beam waveform faithfully. This is essential because some of the experiments conducted at the University of Maryland require time resolved energy spread from the beam. The way to measure the energy spread of the beam is to adjust the retarding voltage. As the retarding voltage is increased, the energy analyzer output becomes smaller. By differentiating the energy analyzer output voltage with respect to the retarding voltage, we can get the beam energy profile information. Figures 9 and 10 show such a case. In Fig. 9, different traces corresponding to different retarding voltages are plotted together. The numbers on the traces correspond to increasing retarding voltages. Note that the beam energy spread information is time resolved. We can see that, at the front end of the beam, the particle energy is larger than that in the main beam due to the longitudinal space-charge force. From this figure, we can construct the beam energy profile by taking the difference of the voltage of traces corresponding to the different retarding voltages. To study the energy spread of the main beam particles, we set the sampling point at the middle of the beam pulse. Figure 10 shows the energy profile. The 


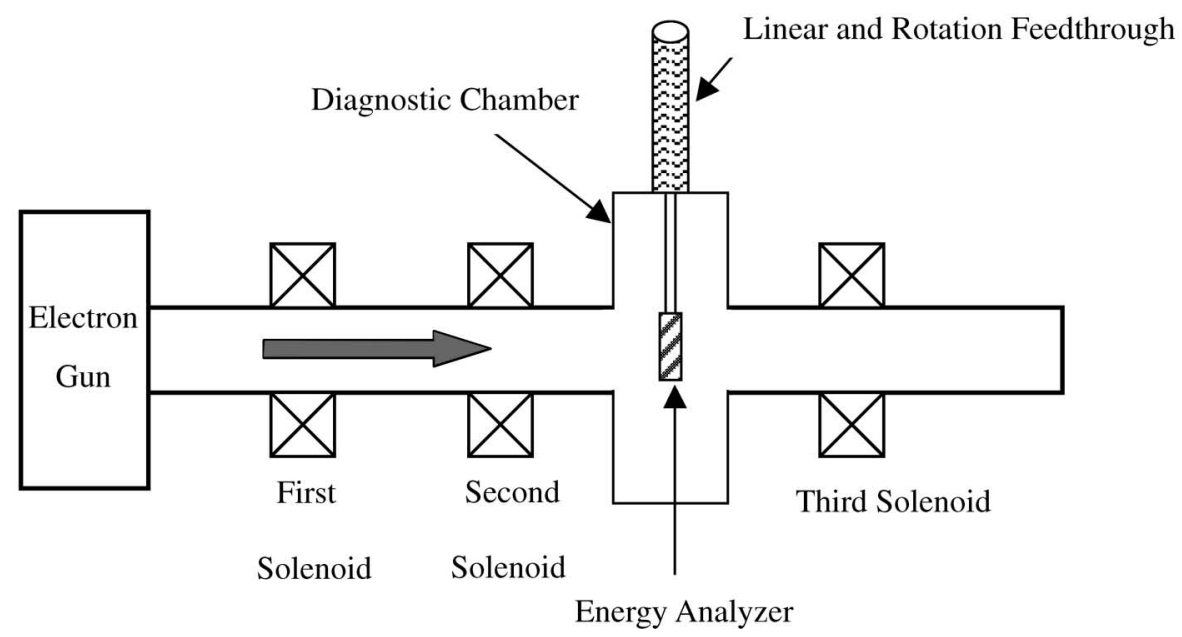

FIG. 6. Experimental setup for the energy analyzer test.

experimental data points and their fit curve are plotted together in this figure. The error bars are also shown here. The magnitudes of the error bars are determined by the signal fluctuations due to the background noise. Note that the energy distribution shown here is asymmetric and has a tail at the high-energy side. More study is underway to explain the source of this tail. In this experiment, the beam energy and current are $2.5 \mathrm{keV}$ and $60 \mathrm{~mA}$, respectively, which is strong space charge dominated. Because of the focusing forces introduced by the cylindrical structure, the defocusing forces due to the space-charge forces and other factors have been well balanced. The measured beam rms energy spread is $1.8 \mathrm{eV}$.

It is interesting to compare the experimental results with the theoretical predictions of the electron beam energy spread. In our example, the beam energy is $2.5 \mathrm{keV}$. The beam is emitted from a cathode with a radius of $5 \mathrm{~mm}$. In the transport system, the average beam radius is around $9 \mathrm{~mm}$. The first term of Eq. (6) gives the energy spread due to the longitudinal-longitudinal effect, which is $0.9 \mathrm{eV}$ in this case. The second term of Eq. (6) gives the rms energy spread due to the Boersch effect, which is $\sim 1.0 \mathrm{eV}$. The

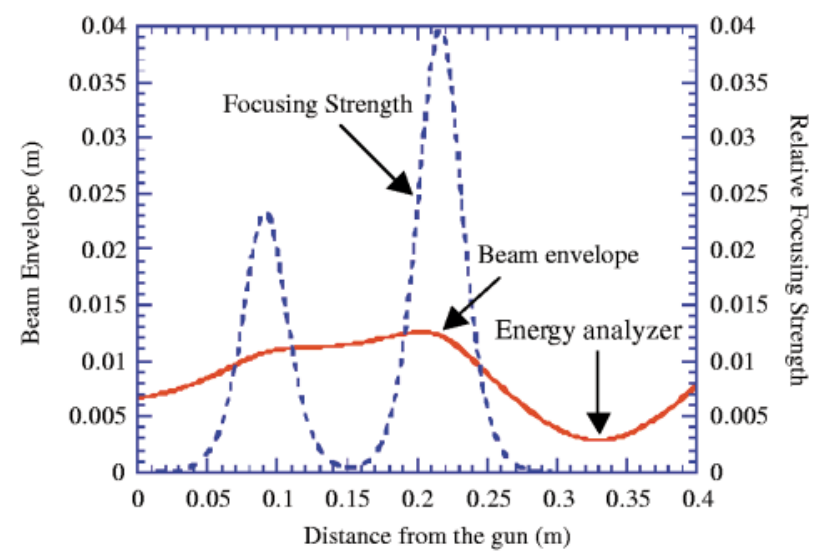

FIG. 7. (Color) Typical beam envelope in the transport line. final energy spread resulting from both effects as given by Eq. (6) is $\sim 1.3 \mathrm{eV}$, which is very close to the experimental result, $1.8 \mathrm{eV}$.

From the comparison, we find that the experimental result is larger than the theoretical predictions. There are several possible reasons for this discrepancy. First, as we explained earlier, the finite resolution of the energy analyzer will contribute to the larger measured beam energy spread. The computer simulation shows that the resolution of this energy analyzer is $0.6 \mathrm{eV}$ for a $2.5 \mathrm{keV}$ beam. In reality, because of the collective space-charge forces and Coulomb scattering of electron beams inside the energy analyzer, the resolution of the energy analyzer might be worse than the computer simulation. A two-dimensional particle-in-cell code simulation is underway to study the behavior of the energy analyzer in more detail. Second, besides these two main energy spread sources mentioned here, there are possible other sources, which may cause an increase of the beam energy spread. In the study reported here, we have considered only the Coulomb scattering theory. However, in space-charge-dominated beams,

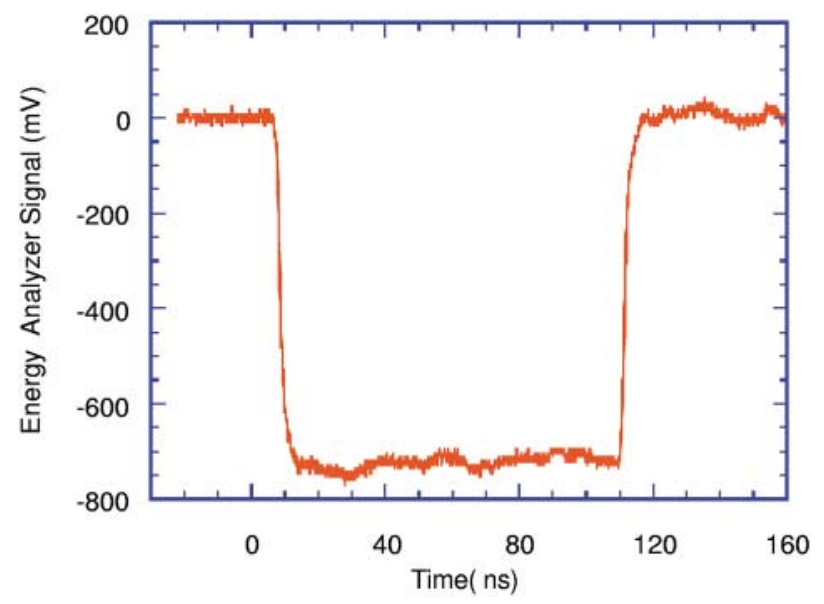

FIG. 8. (Color) Typical energy analyzer output signal. 


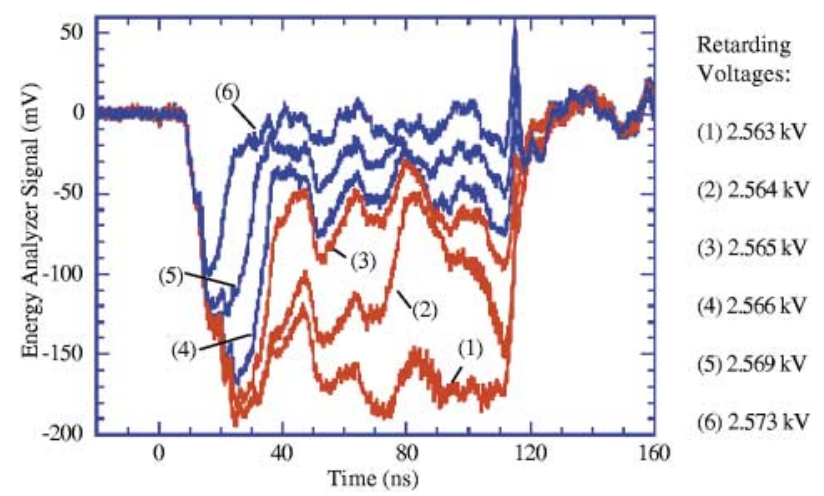

FIG. 9. (Color) Energy analyzer outputs at different retarding voltages.

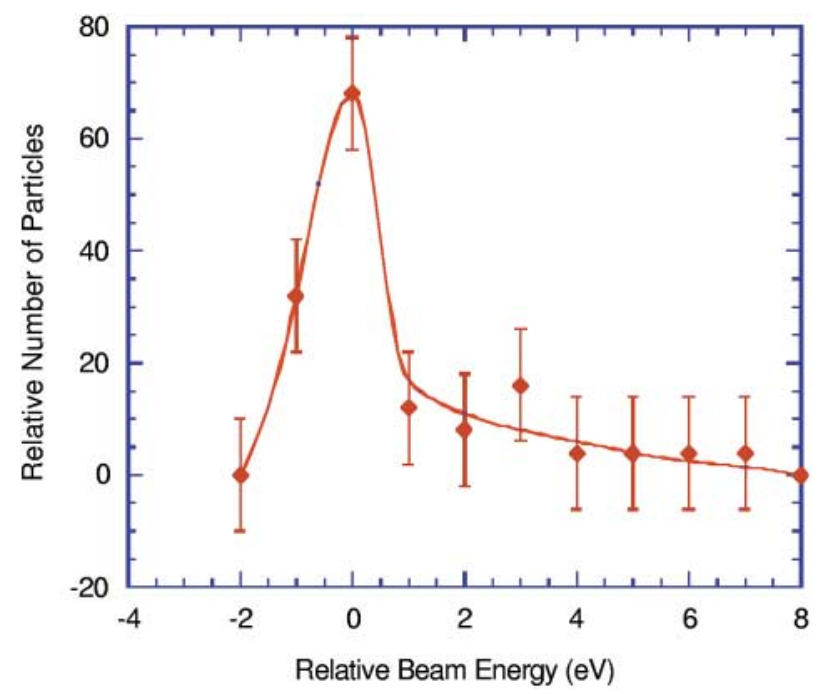

FIG. 10. (Color) Beam energy profile for a beam with energy of $2.5 \mathrm{keV}$ and current of $60 \mathrm{~mA}$. The rms energy width is $1.8 \mathrm{eV}$.

collective instabilities may produce relaxation towards 3D thermal equilibrium. The larger energy spread observed in the experiments may in fact be due to collective instabilities and other effects that will have to be studied in future simulation work. Actually, there are already some simulations in the literature, showing that a beam with a two-temperature anisotropy $\left(T_{\| i} \ll T_{\perp i}\right)$ will develop instability, which may increase the longitudinal energy spread and even significantly deteriorate the beam quality [9-11].

\section{CONCLUDING REMARKS}

A compact high-resolution electrostatic energy analyzer for low-energy electron beams has been designed, simulated, and beam tested. The simulations and beam tests show that the energy analyzer has very good resolution for the energy measurement of space-charge-dominated electron beams in the energy range of several thousand electron volts. The measured beam energy spread is very close to the predictions of a theoretical model based on a longitudinal beam cooling effect, transverse-longitudinal, and longitudinal-longitudinal temperature relaxation effects. A new version of this analyzer, which has higher resolution for the $10 \mathrm{keV}$ UMER beam has been constructed and is currently being tested [12].

Last, it should be noted that our electrostatic analyzer is applicable only to low-energy electron and ion beams. At higher energies, other techniques have to be used to measure the energy spread of the beams.

\section{ACKNOWLEDGMENTS}

This work is supported by DOE Grants No. DEFG0292ER54178 and No. DEFG0294ER40855.

[1] J. G. Wang, H. Suk, and M. Reiser, Phys. Rev. Lett. 79, 1042 (1997).

[2] P. G. O'Shea, M. Reiser, R. A. Kishek, S. Bernal, H. Li, M. Pruessner, V. Yun, Y. Cui, W. Zhang, Y. Zou, T. Godlove, D. Kehne, P. Haldemann, and I. Haber, Nucl. Instrum. Methods Phys. Res., Sect. A 464, 646 (2001).

[3] H. Boersch, Z. Phys. 139, 115 (1954).

[4] A. V. Aleksandrov et al., Phys. Rev. A 46, 6628 (1992).

[5] M. Reiser, Theory and Design of Charged Particle Beams (Wiley, New York, 1994).

[6] J. A. Simpson, Rev. Sci. Instrum. 32, 1283 (1961).

[7] H. Suk, Ph.D. Thesis, University of Maryland, 1996.

[8] Y. Zou, Ph.D. thesis, University of Maryland, 2000.

[9] A. Friedman, D. P. Grote, and I. Haber, Phys. Fluids B 4, 2203 (1992).

[10] I. Haber, D. A. Callahan, A. Friedman, D. P. Grote, and A. B. Langdon, Fusion Eng. Des. 32, 169-174 (1996).

[11] I. Haber, D. A. Callahan, A. Friedman, D. P. Grote, and S. M. Lund, Nucl. Instrum. Methods Phys. Res., Sect. A 415, 405 (1998).

[12] Y. Cui, A. Valfells, S. Bernal, M. Reiser, P. G. O'Shea, R. A. Kishek, V. Yun, and N. Rahimi, in Proceedings of the 2001 Particle Accelerator Conference, Chicago, IL, edited by Peter W. Lucas and Sara Webber (IEEE, Piscataway, NJ, 2001). 